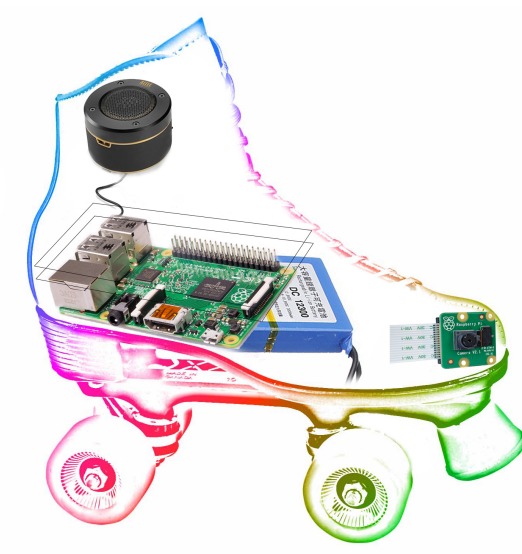

Figure 1: Robot Skate prototype sketch

\title{
'The ghosts of roller disco', a choreographed, interactive performance for robotic roller skates.
}

\author{
Wade Marynowsky** \\ Creativity and Cognition Studios \\ School of Computer Science \\ University of Technology Sydney \\ Sydney, Australia \\ wade.marynowsky@uts.edu.au
}

\section{Angelo Fraietta}

Interactive Media Lab

UNSW Art and Design

University of New South Wales

Sydney, Australia

\author{
Sam Ferguson \\ Creativity and Cognition Studios \\ School of Computer Science \\ University of Technology Sydney \\ Sydney, Australia \\ Samuel.Ferguson@uts.edu.au
}

\author{
Oliver Bown \\ Interactive Media Lab \\ UNSW Art and Design \\ University of New South Wales \\ Sydney, Australia
}

\section{ABSTRACT}

The project investigates how interactions with complex (biologically inspired swarming) behaviors of multiple robots are understood by human participants within a performative and dramaturgical system. Nonanthropomorphic robots in the form of roller skates are used in innovative ways by

Permission to make digital or hard copies of part or all of this work for personal or classroom use is granted without fee provided that copies are not made or distributed for profit or commercial advantage and that copies bear this notice and the full citation on the first page. Copyrights for third-party components of this work must be honored. For all other uses, contact the owner/author(s)

TEI '20, February 9-12, 2020, Sydney, NSW, Australia

() 2020 Copyright held by the owner/author(s).

ACM ISBN 978-1-4503-6107-1/20/02.

https://doi.org/10.1145/3374920.3375284 


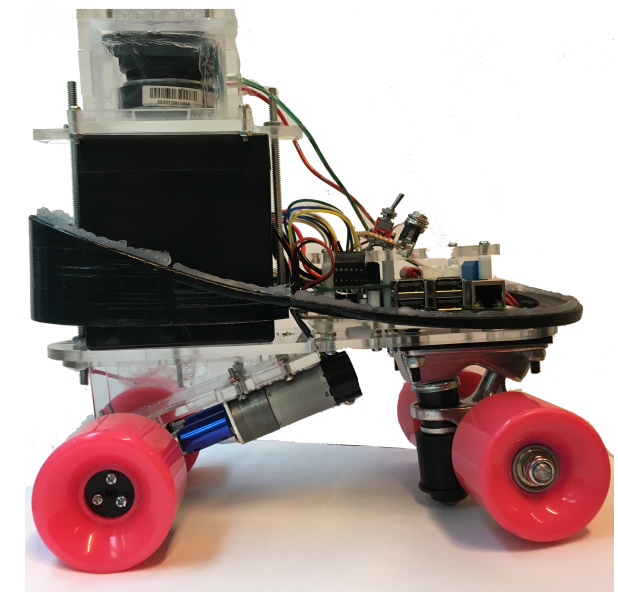

Figure 2: Robo Skate Prototype1 creating social formations from their movements, for example a leader and followers in a conga line. Synchronized audio signals and speech-like sonic structures are used in innovative ways by influencing and engaging the participant's interactions with the robots. Localization data of the robots in space is mapped to control the surround sound and lighting within the space. This is used to enhance audience immersion and engagement within the interactive performance work.

\section{CCS CONCEPTS}

- Human-centered computing $\rightarrow$ Ubiquitous computing; • Applied computing $\rightarrow$ Media arts.

\section{KEYWORDS}

Human-Robot Interaction, indoor-localisation, swarm behaviours, cognitive responses to robotic movement, sound and light.

\section{ACM Reference Format:}

Wade Marynowsky, Sam Ferguson, Angelo Fraietta, and Oliver Bown. 2020. 'The ghosts of roller disco', a choreographed, interactive performance for robotic roller skates.. In Fourteenth International Conference on Tangible, Embedded, and Embodied Interaction (TEI '20), February 9-12, 2020, Sydney, NSW, Australia. ACM, New York, NY, USA, 7 pages. https://doi.org/10.1145/3374920.3375284

\section{INTRODUCTION}

Advances in high-accuracy, low-latency, indoor localisation systems open up many new possibilities for interactions with robots. This project utilises these new capabilities to investigate and simulate biologically inspired swarming behaviours within the domain of Human-Robot Interaction.

Reynolds (1987) [17] pioneered an influential approach to the computer simulation of natural biological flocking and swarming processes. Reynolds's 'boids' algorithm is based on pragmatically very simple behaviours that when combined produce complex results. This has inspired numerous related creative works, for example applying 'boids' to the spatialization of granular audio [10]. Implementing swarms in mobile robots involves additional significant challenges of navigation, planning, sensing and control, but has been demonstrated by Vijay Kumar with his autonomous aerial vehicles [11]. Zooids: Building blocks for swarm user interfaces, is another example of tangible robotic swarms [12]. However, these examples exist in the context of computer science and novel interfaces whilst this research is situated in the context of media arts, which combines art, science and technology in innovative ways. Robotics in the context of media arts has a rich history which stems from experimental art movements such as Fluxus and Performance Art. Pioneering artists of robotic art include: Jean Tinguely; Edward Ihnatowicz and Stelarc to name a few. For further reference see the book; Robots and Art, Exploring an Unlikely Symbiosis [7] and the paper, The origin and development 


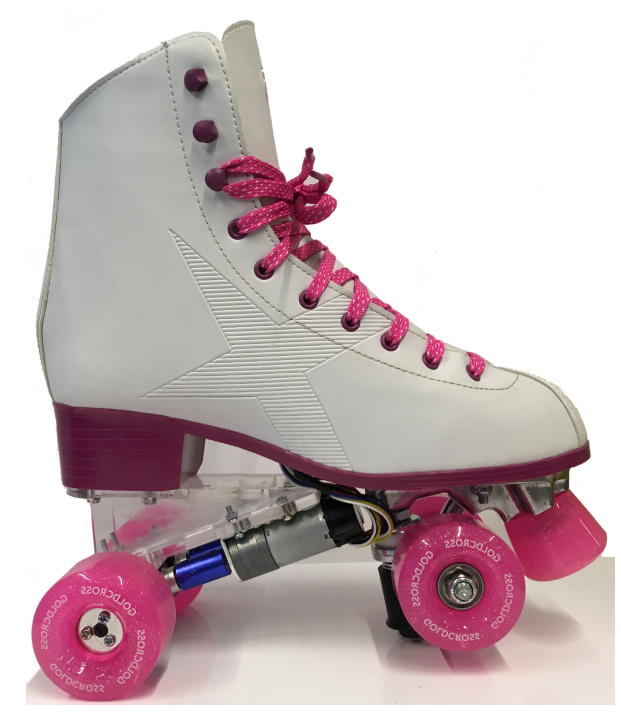

Figure 3: Robo Skate current version of robotic art by Eduardo Kac [9]. Specific to this research is the notion of the robotic multiple and the swarm like behaviours that can emerge from the swarm's collective artificial intelligence.

Bown and Ferguson $[1,3,4]$ define "media multiplicities" as media artworks that consist of multiple interacting devices, connected via digital networks. Increasingly, cheap and flexible embedded systems are making the creation of media multiplicities easier. In robotic form, such multiplicities have the potential to create rich, dramatic, narrative interactive experiences for multiple audience members. In Wade Marynowsky's creative robotics project, Robot Opera, 2015[14] he has established an initial conceptual framework for robotic performance leading to initial insights into robotic performance agency and dramaturgy. It was found that non-human performers could engage audiences in dramaturgical contexts across a sustained performance work of 35 minutes. A secondary finding was that audience members tended to anthropomorphise robot agents and projected social formations (eg. leaders and followers) and hierarchies onto them. Audio signals, speech-like sonic structures and recognizable vocal gestures were seen to influence this strongly as did algorithmic movement interactions with audience members. However, the choreographed sequences within Robot Opera were not completely autonomous and needed additional human input, meaning that complex choreography was limited by the lack of accurate positioning.

\section{AIMS}

This project aims to understand how high accuracy measurements of a group of robot's positions within space can allow for greater choreographic control of the robot swarm in a dynamic interactive context. We also aim to explore how complex behaviours of multiple robotic devices are understood by human participants in the performance, and how they are engaged or influenced by these behaviours. The objective of this project is to develop a system that can operate over a sustained performance or installation work without the need for human supervision, and which can respond interactively to human participation.

\section{TECHNOLOGY}

Hardware: The robotic roller skate (Robo Skate) consists of a CAD designed and laser cut acrylic base. Mounted to the undercarriage are motors retrofitted with real roller skate wheels. To mount the real skate wheels we sourced couplings, right-angle gearboxes and wheel hubs, which secure the skate wheels to the motors. Each motor includes a rotary encoder that facilitates instantaneous detection of motor speed and direction. Inside the boot of the Robo Skate is a battery and a purpose built printed circuit board that functions as both a motherboard for mounting and connecting the other electronics components, and as a Raspberry $\mathrm{Pi}(\mathrm{Pi})$ extension board known as Hardware At Top (HAT) [15]. The HAT features ten I2C ports, an inertial measurement unit, two pairs of GPIO ports for decoding the rotary encoders, and a PCM based digital audio amplifier. Additionally, the 
HAT features a high power MOSFET power distribution circuit to accommodate for the high current requirements of the motors. The $\mathrm{Pi}$ and the ThunderBorg motor controller, commercially available by PiBorg, are securely mounted onto the HAT, providing a single compact unit capable of tolerating mechanical stress to which the units will be subjected. Mounted to the top of the unit is a speaker inside an acrylic box, which enables the networked control of audio.

For the indoor localisation of the Robo Skates we leverage an Ultra Wide Band (UWB), Time Difference Of Arrival (TDOA) system developed by Pozyx. The hardware system consists of multiple 'tags', which broadcast messages including the tags ID and $\mathrm{x}$, $\mathrm{y}$ data. These are received by four 'anchors' (mounted in the ceiling), with small differences in time. From these differences, the central server can calculate the position of the 'tag'. The central server utilises a cloud based web interface for settings configuration and the visualisation of the tags within the space.

Software: The data is subscribed to via an MQTT stream over Wifi and send back to the Pi. The data of a numbered tag is assigned to the specific mac address of a Pi and is used to update the current position. The current $z, y$ positions are measured in relation to the on board gyroscope and motors encoder data which updates a proportional, integral and derivative (PID) controller. The PID manages the differential drive of the motors steering it to the desired position in space. The software was developed in JAVA, and implements HappyBrackets [2,5], an open source toolkit for the Internet of Things (IoT) with powerful audio capabilities. Moreover, HappyBrackets facilitates integration and control of multiple processes, and the development of libraries using multiple programming languages without requiring the artist to switch between different work space environments [6].

\section{EXPANDED BODIES}

Interactive audiovisual works have demonstrated that audience engagement can be sustained in mixed reality artworks, for example, Footfalls (2006)[13], by Golan Levin and Zachary Lieberman an interactive audiovisual installation in which the stomping of the visitor's feet creates cascading avalanches of bouncy virtual forms, using particle systems. However, to date, there has been very limited interactive performance works based on multiple autonomous swarming robots. This research seeks to fill this gap by providing a platform for the exploration of a wider range of interactive experiences using swarm like behaviours.

A series of behaviours have been programmed to simulate human-like figure skating maneuvers, for example, Figure 8's, spins, and circle work. The robots also work together by following each other, mimicking each other and dancing against each other. These formations are choreographed to match the system to the real world, enabling the audience a way to understand the algorithmic movement. These behaviours are meant to entice beauty through our love of geometry and deep rooted connections to symmetry. Whilst swarming behaviours, particularly around humans would inevitably seem creepy, like a swarm of bees readying to sting, The ghosts of roller disco are only cute 

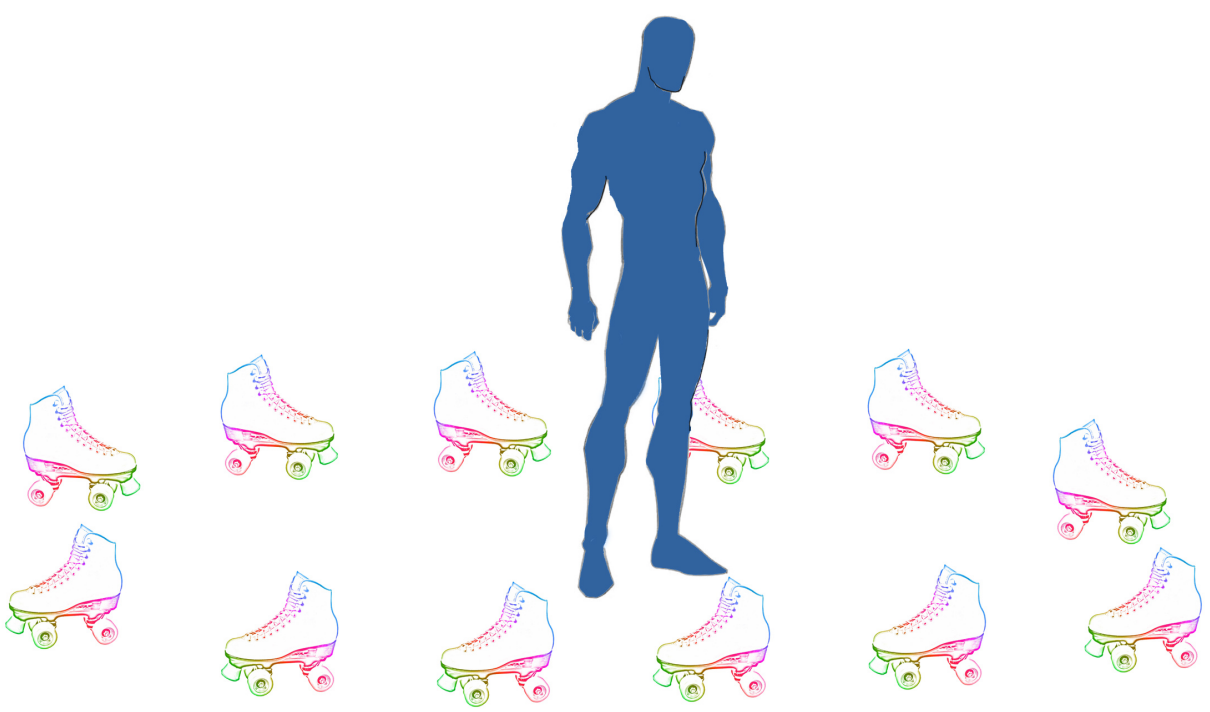

Figure 4: The Robot Skate swarm sketch

robotic roller skates programmed to have minds of their own. The ghosts of roller disco expand our bodies in the form of embodied, and disembodied invisible feet inside roller skate boots. By using the familiar, retro, 80's roller skates the audience response is not threatening or uncanny as with many other, more humanoid robots (such as Geminoid [16] or Sophia [18]), but focused on their own nostalgic reference of roller skating. This is further enhanced by the sound track and sonic structures which recall 80's roller disco music.

\section{END NOTE}

RoboCup founded in 1996 [8] has so far mainly focused on robotic soccer. We propose that robotic art in the form of robotic figure skating, be one of the categories for the next robot Olympics. 


\section{ACKNOWLEDGEMENTS}

Artist: Wade Marynowsky, Hardware: Angelo Fraietta, Brendan Lamb, Nicholas Welsh, Software: Michael Gratton, Sam Ferguson, Alex McClung, Angelo Fraietta, Sound Design: Oliver Bown, Wade

Marynowsky. This project has been assisted by the Australian Government through the Australia Council, its arts funding and advisory body.

\section{REFERENCES}

[1] Oliver Bown and Sam Ferguson. 2018. Understanding media multiplicities. Entertainment Computing 25 (2018), 62-70.

[2] Oliver Bown, Angelo Fraietta, Samuel Ferguson, Lian Loke, and Liam Bray. 2019. Facilitating Creative Exploratory Search with Multiple Networked Audio Devices Using HappyBrackets. In International Conference on New Interfaces for Musical Expression (NIME-2019). Federal University of Rio Grande do Sul, 286-291.

[3] S. Ferguson, A. Rowe, O. Bown, L. Birtles, and C. Bennewith. 2017. Networked pixels: Strategies for building visual and auditory images with distributed independent devices. In C and C 2017 - Proceedings of the 2017 ACM SIGCHI Conference on Creativity and Cognition. https://doi.org/10.1145/3059454.3059480

[4] Sam Ferguson, Anthony Rowe, Oliver Bown, Liam Birtles, and Chris Bennewith. 2017. Sound Design for a System of 1000 Distributed Independent Audio-Visual Devices. In NIME 2017 Proceedings of the International Conference on New Interfaces for Musical Expression. https://doi.org/10.1002/jlcr.3207

[5] Angelo Fraietta and Oliver Bown. 2019. Creating a Sonified Spacecraft game using HappyBrackets and Stellarium. In Procedings of the 17th Linux Audio Conference (LAC-19). CCRMA, Stanford University, USA, 1-7.

[6] Angelo Fraietta, Oliver Bown, Samuel Ferguson, Sam Gillespie, and Liam Bray. 2020. Rapid Composition for Networked Devices with HappyBrackets. Computer Music Journal 43, 1 (2020). To appear.

[7] Damith Herath, Christian Kroos, et al. 2016. Robots and Art: Exploring an Unlikely Symbiosis. Springer.

[8] Fabrice Jumel. 2019. Advancing Research at the RoboCup@ Home Competition [Competitions]. IEEE Robotics \& Automation Magazine 26, 2 (2019), 7-9.

[9] Eduardo Kac. 2001. The origin and development of robotic art. Convergence 7, 1 (2001), 76-86.

[10] David Kim-Boyle. [n.d.]. Spectral and Granular Spatialization with Boids.

[11] Alex Kushleyev, Daniel Mellinger, Caitlin Powers, and Vijay Kumar. 2013. Towards a swarm of agile micro quadrotors. Autonomous Robots 35, 4 (2013), 287-300.

[12] Mathieu Le Goc, Lawrence H Kim, Ali Parsaei, Jean-Daniel Fekete, Pierre Dragicevic, and Sean Follmer. 2016. Zooids Building blocks for swarm user interfaces. In Proceedings of the 29th Annual Symposium on User Interface Software and Technology. ACM, 97-109.

[13] Golan Levin and Zachary Lieberman. 2004. In-situ speech visualization in real-time interactive installation and performance. In NPAR, Vol. 4. 7-14.

[14] Wade Marynowsky, Julian Knowles, and Andrew Frost. 2015. Robot opera: a Gesamtkunstwerk for the 21st century. In International Workshop on Cultural Robotics. Springer, 143-158.

[15] Simon Monk. 2016. Raspberry Pi cookbook: Software and hardware problems and solutions. "O’Reilly Media, Inc.", Sebastopol, CA, USA.

[16] Shuichi Nishio, Hiroshi Ishiguro, and Norihiro Hagita. 2007. Geminoid: Teleoperated android of an existing person. In Humanoid robots: New developments. IntechOpen.

[17] Craig W Reynolds. 1987. Flocks, Herds, and Schools: A Distributed Behavioral Model. Computer Graphics 21, 4 (1987), $25-34$ 
[18] Chris Weller. 2017. Meet the first-ever robot citizen, a humanoid named Sophia that once said it would destroy humans. Business Insider (2017), 7-10. 